\title{
High Temperature Structure in Cool Binary Stars
}

\author{
A. K. DUPREE, N. S. BRICKHOUSE, AND G. J. HANSON
}

Harvard-Smithsonian Center for Astrophysics, 60 Garden Street, Cambridge, MA 02138 USA

Strong high temperature emission lines in the EUVE spectra of binary stars containing cool components (Alpha Aur [Capella], 44 $\iota$ Boo, Lambda And, and VY Ari) provide the basis to define reliably the differential emission measure of hot plasma. The emission measure distributions for the short-period ( $\mathrm{P} \leq 13 \mathrm{~d}$ ) binary systems show a high temperature enhancement over a relatively narrow temperature region similar to that originally found in Capella (Dupree et al. 1993). The emission measure distributions of rapidly rotating single stars 31 Com and AB Dor also contain a local enhancement of the emission measure although at different temperatures and width from Capella, suggesting that the enhancement in these objects may be characteristic of rapid rotation of a stellar corona. This feature might be identified with a (polar) active region, although its density and absolute size are unknown; in the binaries Capella and VY Ari, the feature is narrow and it may arise from an interaction region between the components.

\section{Introduction}

The emission measure defined by the well-exposed EUV spectra of the binary system Capella (G8 III + G0 III) surprisingly revealed a narrow high temperature "bump" (Dupree et al. 1993) occurring at $T=6.3 \times 10^{6} \mathrm{~K}$. The feature is continuously present irrespective of the orbital phase of the system (Dupree et al. 1994; 1995), and may arise from a continuously visible hot region on one component, or from an interacting region between the stars. The distribution of the high temperature emission measure in the Capella system differs from the broad emission measure distribution found in a solar active region (see Brickhouse et al. 1995). We investigate whether this feature is characteristic of binary systems and whether rotation controls its presence.

\section{Spectra and the Emission Measure Distribution}

$E U V E$ spectra from our Guest Observer program and from the EUVE Science Data Archives were obtained for four binary systems (VY Ari, Capella, $44 \iota$ Boo, $\lambda$ And) and two rapidly rotating single stars (AB Dor and 31 Com). EUV spectra were extracted from the images and calibrated in a uniform manner. The reduced spectra are shown in Fig. 1. In spite of the varying physical characteristics of the stars, there are similarities among the spectra. The resonance line of He II ( $\lambda 304$ ) dominates the MW spectra; strong resonance lines of iron dominate the SW spectra. However, the relative strengths of the lines differ among these objects, and so require detailed evaluation of the emission measure. It is the strength of the Fe XVIII and Fe XIX transitions relative to $\mathrm{Fe} X X$, XXIII that controls the presence of the locally enhanced emission measure (the "bump") in Capella.

Signal to noise ratios were evaluated at the EUVE spectral resolution, and only the strongest features $(S / N \approx 9-15)$ were selected to determine the emission measure. Correction of the observed fluxes for interstellar absorption was made with $N_{\mathrm{H}}$ from Table 1. The H/He abundance ratio was set to 11.6 (Kimble et al. 1993) and helium was assumed to be neutral. Assumption of higher values for $N_{\mathrm{H}}$ would strengthen the 


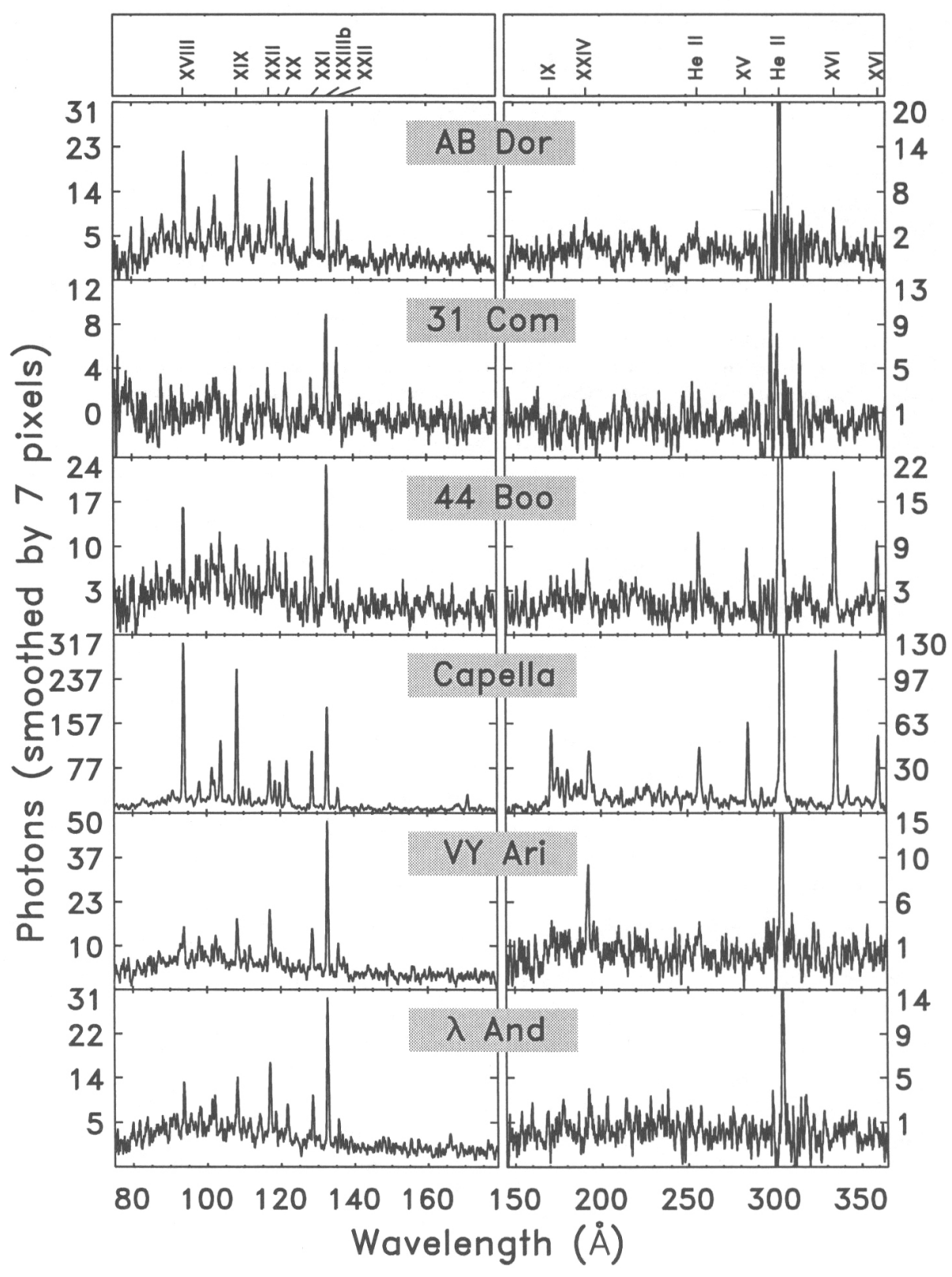

FIGURE 1. EUVE spectra in the SW (left panel) and MW (right panel) band for the rapidly rotating single stars AB Dor and 31 Com and the binaries 44 Boo, Capella, VY Ari, and $\lambda$ And. Species of Fe are marked by ionization stage. Fe XXIII $(\lambda 132.8)$ is blended with Fe XX. 
Table 1. Parameters of Target Stars

$\begin{array}{lrccccc}\text { HD } & \text { Star } & \text { Sp. Type/Lum. } & \begin{array}{c}\text { Porbital } \\ \text { (days) }\end{array} & \begin{array}{c}\mathrm{P}_{\text {phtm. }} \\ (\text { days })\end{array} & \begin{array}{c}N_{\mathrm{H}} \\ \left(\mathrm{cm}^{-2}\right)\end{array} & \begin{array}{c}\text { SW Exp } \\ (\mathrm{s})\end{array} \\ 17433 & \text { VY Ari } \dagger & \text { K3-4V-IV } & 13.208 & 16.64 & 1.0 \times 10^{18} & 168557 \\ 34029 & \alpha \text { Aur } \ddagger & \text { G0 III/G8 III } & 104.0 & 8 / 80 & 1.8 \times 10^{18} & 281571 \\ 36705 & \text { AB Dor } & \text { K1 III } & \ldots & 0.514 & 1.0 \times 10^{18} & 166460 \\ 111812 & 31 \text { Com\| } & \text { G0 III } & <6 & \ldots & 1.0 \times 10^{18} & 84503 \\ 133640 & 44 \iota \text { Boot } \dagger & \text { G0V } & 0.268 & \ldots & 1.0 \times 10^{18} & 104145 \\ 222107 & \lambda \text { And } \dagger & \text { G8IV-III } & 20.52 & 53.95 & 4.0 \times 10^{18} & 105701\end{array}$

t RS CVn-type binary.

$\ddagger$ RS CVn-type binary; EUVE spectra of Capella taken at several phases were averaged together.

T Rapidly rotating single star.

II 31 Com is a rapidly rotating single star thought to be a Hertzsprung Gap giant. The "orbital" period was evaluated from the $v \sin i$ value of $77 \mathrm{~km} \mathrm{~s}^{-1}$, assuming a radius of $9 R_{\odot}$.

i† W UMa-type contact binary.

Fe XVI emission ( $\lambda 336$ ) relative to the Fe XV emission ( $\lambda 284)$ where observed, but leave the fluxes in the short wavelength region $(\lambda 85-\lambda 170)$ essentially unchanged.

The emission measure distribution is determined iteratively, by fully integrating the line emissivities through a trial emission measure distribution, and comparing the predicted and observed line fluxes. There is no restriction on the "smoothness" of the emission measure distribution such as occurs with spectral fitting procedures; moreover the fit is not degraded by bins containing noise or weak signals. Only strong lines are considered in evaluating the emission measure. We require that these fluxes agree within acceptable uncertainties which in this case is better than a factor of two. Detailed current models for all iron ions (Brickhouse et al. 1995) were used. Results for $\lambda$ And are taken from another publication (Hanson et al. 1995) where fluxes of ultraviolet lines measured with $I U E$ were included so that the emission measure below $2 \times 10^{5} \mathrm{~K}$ is well-defined.

\section{Conclusions}

All of the target stars exhibit a range of high ion stages indicating a generally continuous distribution of temperature. The spectral resolution of $E U V E$ demonstrates that two-temperature fits which rely on energy band measures do not represent these stellar atmospheres. For the weaker spectra, the strongest observable lines arise from high stages of ionization (Fe XVIII ... XXIII), the Fe XV $(\lambda 284)$ and Fe XVI ( $\lambda 336$ and $\lambda 361)$ emission is not prominent, and the emission measure can only be reliably defined in a restricted temperature range.

Inspection of the emission measure distributions contained in Fig. 2 and 3 shows that the Capella "bump" is not unique. The binary systems (VY Ari, 44 $\iota$ Boo) clearly show the enhanced emission measure distribution similar to Capella. The appearance of the emission measure enhancement is most pronounced in binary systems with short orbital period ( $\leq 13$ days) and/or high rotational velocity. The orbital period of Capella is 104 days, however the hot component of Capella is rotating rapidly with a period of $\approx 8$ days (Fekel et al. 1986) so that the system may be classified as a rapid rotator for these

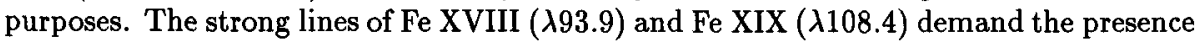




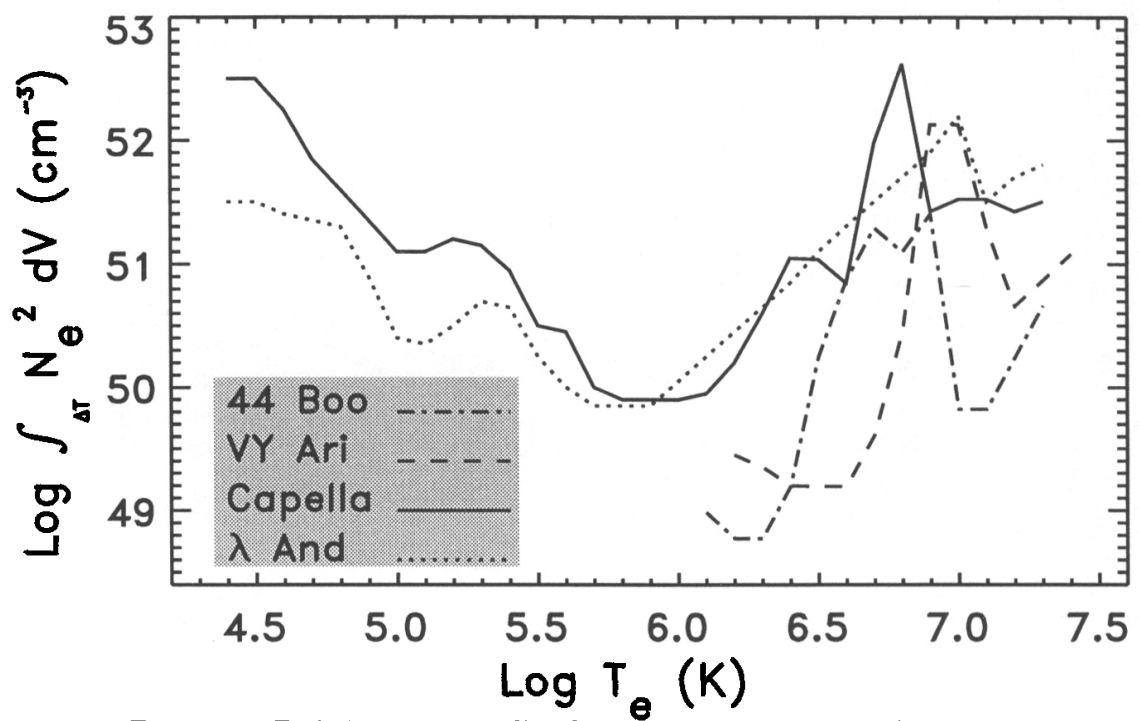

Figure 2. Emission measure distribution determined for 4 binary systems.

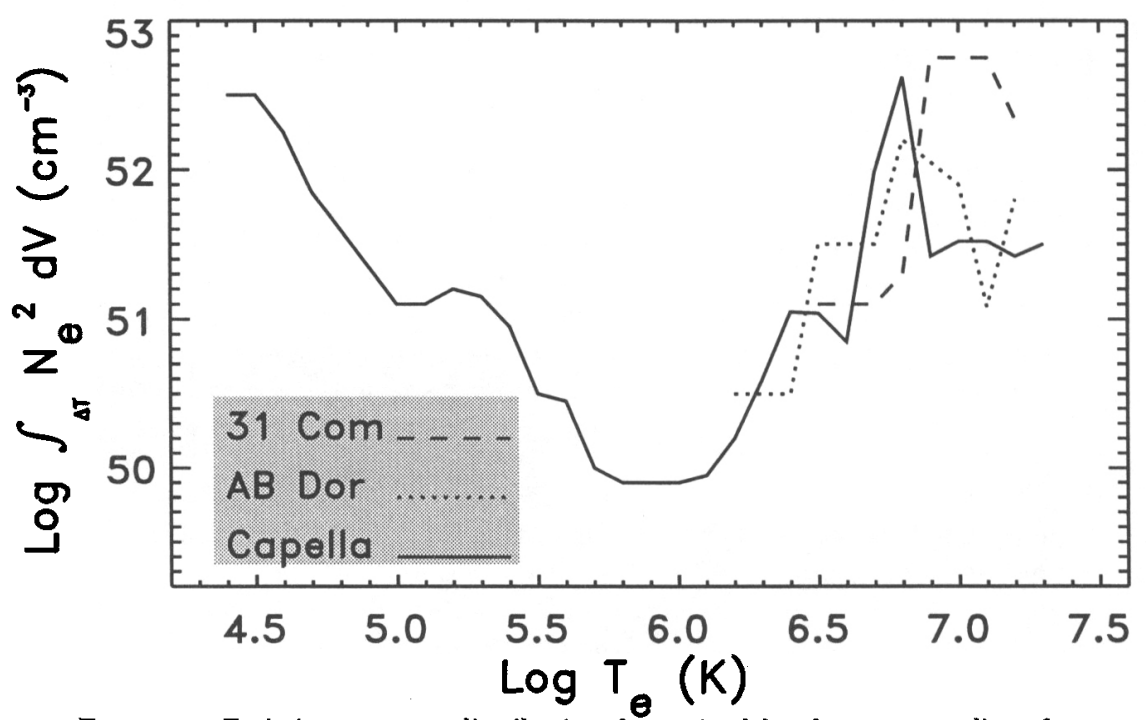

FIGURE 3. Emission measure distribution determined by the strongest lines for two rapidly-rotating single stars ( $31 \mathrm{Com}$ and $\mathrm{AB}$ Dor) and the binary system Capella.

of an increased emission measure in Capella for their production. That $44 \iota$ Boo would have a similar feature is apparent from inspection of its SW spectrum; the enhancement is broader in temperature than the Capella bump. The SW spectrum of VY Ari shows an exceptionally strong feature of Fe XX, XXIII ( $\lambda 132.8)$ relative to the Fe XVIII and XIX transitions; thus the local enhancement of the emission measure occurs at a temperature higher by $0.2 \mathrm{dex}$, namely $T(\mathrm{~K})=7.0 \mathrm{dex}$. The long period system, $\lambda$ And has a much 
less well defined emission measure enhancement (Hanson et al. 1995). The slopes of the emission measures with temperature are consistent with models of magnetic loops or arcades.

Two single stars can address the question of whether the "bump" is unique to binaries. The rapidly-rotating single giant $31 \mathrm{Com}$ is of particular interest because it lies in the Hertzsprung gap (Wallerstein et al. 1994). Since the hot rapidly-rotating secondary in the Capella system is believed (Pilachowski \& Sowell 1992) to be passing through the Hertzsprung gap too, 31 Com might prove helpful in disentangling the composite EUV spectrum of Capella. The star $31 \mathrm{Com}$ has a broad enhancement required by the dominant Fe XXII and Fe XXIII transitions, at a temperature higher (7.0 dex) than the Capella feature. Such a broad hot component is not found in the Capella emission measure. AB Dor, another rapidly-rotating single giant shows a broad enhancement, also different from Capella. Thus, rapidly-rotating single stars have "bumps" but they appear different from those in Capella and VY Ari.

Rotation appears to be a significant physical parameter in producing an enhanced emission measure feature in cool star atmospheres. This feature is generally present in those systems with periods $\leq 13$ days. However the temperature of the maximum and the width and strength of the enhancement differ from star to star. It is plausible to associate this feature with magnetic structures in a rapidly-rotating corona, in which case it could be dense and of small scale; however, in the binary systems Capella and VY Ari, a narrow enhancement (of unknown density) is found. It may be that such a feature results from interaction between the components. Binaries of longer period, represented by $\lambda$ And and $\sigma$ Gem (Hanson et al. 1995) do not show such well-defined enhancements in the emission measure, although they possess equally hot plasma. However longer EUV exposures are needed on many of these stars to detect weaker species and to define the atmospheric structure over a wider temperature range.

This work is partly supported by NASA NAG5-2330 to the Smithsonian Institution.

\section{REFERENCES}

Brickhouse, N. S., RAymond, J. C., \& SMIth, B. W. 1995, New Model of Iron Spectra in the Extreme Ultraviolet and Application to SERTS and EUVE Observations of a Solar Active Region and Capella, ApJS, 97, 551

Dupree, A. K., Brickhouse, N. S., Doschek, G. A., Green, J. C., \& Raymond, J. C. 1993, The Extreme Ultraviolet Spectrum of Alpha Aurigae Capella, ApJ, 418, L41-L44

Dupree, A. K., Brickhouse, N. S., DoscheK, G. A., Hanson, G. J., \& Raymond, J. C. 1994, EUVE Spectra of Alpha Aurigae Capella, at Different Phases, BAAS, 26, 864

Dupree, A. K., Brickhouse, N. S., \& Hanson, G. J. 1995, Inhomogeneity of Coronal Structures in Capella, in prereparation

FeKel, F. C., Moffett, T. J., \& HeNRY, G. W. 1986, A Survey of Chromospherically Active Stars, ApJS, 60, 551

Hanson, G. J., Brickhouse, N. S., \& Dupree, A. K. 1995, EUVE Spectra of Lambda Andromedae and Sigma Geminorum, ApJ, submitted

KnMBLe, R. PLUS, 13 AUTHORS 1993, Extreme Ultraviolet Observations of G191-B2B and the Local Interstellar Medium with the Hopkins Ultraviolet Telescope, ApJ, 404, 663

Prtachowski, C. A., \& Sowell, J. R. 1992, The Lithium Abundance of the Capella Giants, AJ, 103, 1668

Wallerstein, G., Bohm-Vitense, E., Vanture, A. D., \& Gonzalez, G. 1994, The Lithium Content and Other Properties of F2-G5 Giants in the Hertzsprung Gap, AJ, 107, 2211 